\title{
Towards Deployment of the Remote Instrumentation e-Infrastructure (in the Frame of the DORII Project)
}

\author{
Alexey Cheptsov ${ }^{1}$, Rainer Keller ${ }^{1}$ \\ Roberto Pugliese ${ }^{2}$, Milan Prica ${ }^{2}$, Andrea Del Linz ${ }^{2}$ \\ Marcin Plociennik ${ }^{3}$, Marcin Lawenda ${ }^{3}$ and Norbert Meyer ${ }^{3}$ \\ ${ }^{1}$ High-Performance Computing Center, University of Stuttgart, Germany \\ ${ }^{2}$ ELETTRA, Sincrotrone Trieste SCPA, Italy \\ ${ }^{3}$ Poznań Supercomputing and Networking Center, Poland
}

(Received: 18 December 2008; published online: 25 March 2009)

\begin{abstract}
While the majority of e-Infrastructures that are set up within the European Research Area by such projects as EGEE, DEISA etc. are basically focused on providing the high-performance computing support for scientific applications, a diverse set of the scientific communities coming from various fields (e.g. earthquake, environmental science, experimental science communities) develop and operate experimental equipment and remote instrumentation that have not been integrated yet or only partially integrated within the European Grid e-Infrastructure. The Deployment of Remote Instrumentation Infrastructure (DORII) project aims at setting up an advanced Grid-based e-Infrastructure specifically oriented to the support of remote instrumentation devices on Grid extending the level of scientific instruments' exploitation. The paper highlights the main application areas and usage scenarios, key tasks of the Remote Instrumentation Infrastructure's deployment and presents the joint research architecture for DORII in terms of advanced middleware solutions addressing the main tasks of identified applications.
\end{abstract}

Key words: remote instrumentation, e-Infrastructure, middleware, Grid

\section{INTRODUCTION}

In recent years the progress of the high-performance computing has enabled the deployment of large-scale e-Infrastructures like those promoted by the EGEE [1] and DEISA [2] projects in the European Research Area, OSG [3] in USA or NAREGI [4] in Japan. They provide powerful distributed computing environments facilitating the everyday research done by scientists, in particular in the context of the e-Science collaboration [5]. A major focus of the most set up e-Infrastructures is the support of distributed computation and data-intensive scientific parallel applications. However, in a big variety of e-Science communities (including Environmental Science, Earthquake Engineering, On-Line Data Analysis in Experimental Science) many interests have recently arisen in the establishment of new IT infrastructures for the operational support of scientific applications using the remote instrumentation and experimental laboratory equipment [6-9].
For leveraging grid capabilities for experimental e-Science communities operating remote instruments and sensors, the Remote Instrumentation e-Infrastructure which is being developed in the frame of the EU-funded DORII project is proposed. Extending the level of scientific instruments' exploitation, the Remote Instrumentation e-Infrastructure will enable flexible and unified providing Remote Instrumentation Services (particularly studied and developed in the frame of several previous European projects, such as RinGRID [10] and GRIDCC [11], or CIMA [12] in USA) together with common computing and storage resources. On the foundations of these projects, DORII [13] is setting up a new e-Infrastructure addressing different areas of the remote instrumentation applications support on the Grid. This will be done in a way that would allow reusing procedures, middleware and services developed for further deployment outside the project.

The goal of the presented paper is to highlight basic aspects of the architecture design that was proposed for the 
DORII project, particularly with respect to requirements of specific application fields, in terms of advanced middleware solutions for e-Science. The paper is organized as follows. We first discuss aims and objectives of the Remote Instrumentation e-Infrastructure's deployment. The potential contribution that the e-Infrastructure can make towards providing an efficient and safety supporting mechanism for the remote instrumentation is described as well. We also identify requirements of applications and instrumentation devices of the DORII scientific communities and describe application areas of the deployed Remote Instrumentation e-Infrastructure. Finally we present a solution that enables deployment, remote operation and exploitation of scientific instruments and applications taking advantages of best practices of the European Grid e-Infrastructure, as it is proposed in the frame of the DORII project.

\section{TASKS OF THE REMOTE INSTRUMENTATION PROVIDED BY THE E-INFRASTRUCTURE}

Remote Instrumentation Services are an important part of a wide range of grid-based applications. These include oceanographic applications, earthquake engineering, largescale physics experiment devices and many other interdisciplinary e-Science applications. While recent developments in the area of grid technologies have mainly focused on providing a batch access to distributed computing and storage resources, accessing the remote instrumentation and laboratory equipment of scientific applications as a full service component of distributed grid resources is still an imperative requirement for the set up e-Infrastructures. Deployment of a Remote Instrumentation e-Infrastructure enables distributed applications a full range of management, configuration, controlling, monitoring, fine-tuning and other possibilities for their remote instrumentation allowing experiments in various fields of e-Science to be carried out.

However, integrating a wide range of instruments is challenging because of the sheer diversity of instruments, from real-time sensors that continually provide low traffic data streams to devices that are used infrequently and produce large amounts of data. Existing remote instrumentation capabilities of the e-Infrastructure include frameworks that allow performing remote controlling of scientific instruments and acquiring measurements results through a sequence of necessary commands defined by instrumentation's interfaces. However, a Remote Instrumentation e-Infrastructure should provide a much wider functionality in order to:
- extend the usage of grid computing technologies to enable the accessing and controlling of distributed instrumentation devices, integration of instrument and sensors to the Grid;

- provide a set of standard capabilities to perform all the instruments functionality with respect to requirements of e-Science communities;

- construct a suitable abstraction of the remote instrumentation in order to make it available for the e-Infrastructure as a manageable grid-enabled resource;

- present a standard user interface allowing to browse the "distributed laboratory space", choose different pieces of equipment, configure their interconnection, orchestrate experiment executions, collect, process, store and analyze the results, and make them available to e-Science communities through experiment data repositories, established as Digital Libraries;

- allow the user to design complex workflows for controlling instruments providing all necessary submission and monitoring in a real time capabilities.

In order to accomplish these tasks to a full extent, instrumentation devices should become full class members of the Open Grid Service Architecture (OGSA) [14], in the same way as computation and storage resources. The support of the remote instrumentation by the e-Infrastructure poses challenges in terms of both networking and monitoring the different typology of resources and ways to support collective use of all the Remote Instrumentation Services. Test resources of the e-Infrastructure should thereby provide: 1) isolation from and relative independence of the underlying networking infrastructure; 2) services for resource allocation and management; 3) standard user interfaces; 4) non-trivial Quality of Service (QoS) control and QoS-aware workflows; 5) integration in Grid and peer-to-peer overlays. These all can be put in a perspective within the OGSA framework by enhancing the existing Remote Instrumentation Service capabilities in the deployed e-Infrastructure.

\section{THE DORII PROJECT}

DORII [13] started in February 2008 and aims at deploying a new e-Infrastructure for scientific communities that operate the experimental equipment and instrumentation that have not integrated yet or only partially integrated in the European Grid e-Infrastructure. Building on the experience gained in a number of previous and ongoing 
European projects on the remote instrumentation (among others, RINGrid [10] and GRIDCC [11]), interactivity and MPI support of parallel applications (Int.EU.Grid [15]), application development and frameworks (g-Eclipse [16]), virtual laboratories (VLAB [17]) and advanced networking technologies (Geant2 [18]), DORII is currently designing and setting up an extended e-Infrastructure which is focusing on users in three main e-Science areas:

1. Earthquake community (with various sensor networks);

2. Environmental science community (with several geoshared instruments);

3. Experimental physics community (with synchrotron and free electron lasers).

Working closely with end-users, DORII recognized the following strategic goals and objectives: 1) to adopt the e-Infrastructure functionality across selected areas of science and engineering; 2) to deploy and operate the persistent, production quality, distributed instrumentation integrated with the e-Infrastructure; 3 ) to generalize and deploy a framework environment that can be used for fast prototyping.

These objectives will be achieved in DORII in three phases. The first phase is an integration and adaptation of the selected products from previous projects. The second phase is an establishment of an infrastructure for DORII. The applications will be developed on the e-Infrastructure and enhanced by currently provided Remote Instrumentation Services. The third phase is a standardization and deployment of the results to a wider community of e-Science outside the project.

DORII partners have already established a research group inside Open Grid Forum [19], which exactly focuses on topics related with the project, i.e. RISGE - Remote Instrumentation Services in a Grid Environment. RISGE explores issues related to the exploitation of grid technologies for conducting and monitoring measurement tasks and experiments on complex remote scientific equipment. The main purpose of this research group is to bring together various existing approaches in defining remote access interfaces to sophisticated laboratory equipment, as well as to come up with use cases that can dictate the requirements for integrating scientific instruments with the Grid. One of the results of RISGE activity could be the establishment of a working group for the standardization of relevant capabilities.

Accomplishing the defined tasks, DORII will provide following support environments for users of the e-Infrastructure:

- Remote instrumentation environment - includes grid-enabled remote instrumentation and sensor devices. The DORII e-Infrastructure will provide similar concepts and abstractions for different usage scenarios of Remote Instrumentation Services, enabling interoperability and compatibility of the "gridified" instrumentation.

- Application environment - applications of experimental e-Science communities supported by DORII that are being developed and supported by numerous projects and consortia as well as deployment, implementation and instrumentation libraries for them, in particular MPI libraries for parallel applications.

- Middleware environment - consists of core grid services extended by a set of middleware solutions developed and enhanced in DORII (such as Virtual Control Room [20]) for the resource and data management as well as a number of transport, security, and information services.

\section{- Hardware and networking infrastructure.}

At the time of this paper, the established infrastructure consists of 8 grid sites distributing among the partners of the project. The provided grid sites are also a part of the EGEE production infrastructure and part members of the Int.EU.Grid infrastructure. In total, these sites provide 2200 non-dedicated CPUs and several Terabytes storage to the DORII users and their applications. For the initial deployment of the applications a Catch-all Virtual Organization [21] named "vo.dorii.eu" is established. For some project members other VOs (such as "imain", "ihydra" etc.) will be operated as well.

The networking infrastructure for DORII is being deployed, focusing on ensuring high reliability of the provided Remote Instrumentation Services. Local access networks (LANs) will be upgraded to the Gigabit level and, wherever possible, traffic priority mechanisms will be introduced. As an additional service for the applications with high requirements on the networking bandwidth, a Bandwidth on Demand (BoD) as well as IPv6 services might be supported as well.

\section{APPLICATION AREAS OF THE REMOTE INSTRUMENTATION e-INFRASTRUCTURE}

The DORII project will make e-Infrastructures available for various applications operating and using the remote instrumentation. The scientific communities participating in DORII span a significant range of e-Science domains, each posing several challenges to the effective exploitation of provided services and facilities. With regard to 
the main usage scenarios the DORII applications can be classified to the two major categories: applications that provide Remote Instrumentation Services (such as "Online Processing in Experimental Science" working on data acquired in a real time or "Network-Centric Seismic Simulation" processing pre-collected data by using a sensor network) and applications for the post-processing analysis and simulation (such as OPATM-BFM simulation model provided by the application "Oceanographic and Coastal Observation and Modeling").

For each of the application categories there are several differentiated scenarios of the Remote Instrumentation e-Infrastructure's usage, as described in the following:

- Real-time data acquisition and processing Applications for online processing in experimental science cover a wide class of experimental stations in facilities like Synchrotrons and Free Electron Lasers of ELETTRA [22] that produce huge quantities of data. These data need to be analyzed on-line, which requires considerable computing power and often teamwork. The problem is even more difficult considering the increased efficiency of the light sources and detectors. Complex calculations are required to take diffraction images and convert them in a 3D protein structure. Similarly, complex computations are required to calculate tomography and then perform the result analysis. The results of analyses often need to be visualized for a distributed team of experts and used to interactively modify the data collection strategy. Data from instruments and sensors are saved in distributed repositories, computational models are executed, and finally an interactive data mining process is used to extract practical knowledge.

The "Network-Centric Seismic Simulation" application of EUCENTRE [23] aims at performing pseudo-dynamic simulations using sub-structuring. This means that a part of the building being simulated is a "virtual" structure, while another part is a physical specimen placed in a laboratory and equipped with actuators (to apply forces or displacements) and sensors (to measure reactions). The simulation server collects the data provided by the sensors and the calculated response of the virtual building components, putting all together in order to represent this set as a unique structure.

All the applications require both the support of a standard grid computing environment, which is a virtual organization, a set of distributed storage and computing resources and a resource brokering mechanism, a workflow definition and execution environment and the capability to integrate instruments (the detectors) and interactively collaborate in the data analysis process. A QoS handling mechanism is necessary to use effectively the available network structure.

- Post-processing analysis and simulation

Another type of applications that can benefit by using the e-Infrastructure is data post-processing analysis and simulation applications. Whereas the applications operating the remote instrumentation set numerous requirements on servicing and instrumentation capabilities provided by the e-Infrastructure, for data post-processing analysis and simulation applications an important aspect of porting to the Grid becomes getting a high productivity and performance.

OPATM-BFM is an off-line three-dimensional coupled eco-hydrodynamic simulation model used for biogeochemical and ecosystem-level predictions of the Mediterranean Sea developed by OGS [24]. It is the core of a forecasting system embedded in a fully automatic procedure that produces maps of biogeochemical concentrations for the whole Mediterranean basin on a weekly basis. However, the model is expected to deliver additional products and information for different time scales as well as for climatic scenario analyses (multi-decadal period of integration). This constitutes the most considerable limitation of the application scalability.

Together with other analysis and simulation applications, the e-Infrastructure will provide OPATMBFM with a full-range access to the distributed computing and storage resources, allowing to maximize the application performance characteristics. A number of development, maintaining, debugging and performance analysis possibilities will be enabled for the applications as well. Moreover, the instrumentation services provided by the e-Infrastructure will facilitate the coupling with instrumentation applications which provide initial conditions and input data necessary for the simulation and analysis.

Regardless of the highlighted usage scenarios, scientific applications will take advantages of using the DORII e-Infrastructure in terms of offered middleware tools and solutions as well as provided instrumentation services. A flexible environment that DORII will set up will allow to integrate the remote instrumentation into the e-Infrastructure as a full-fledged grid component enabling a full range of provided Remote Instrumentation Services for the scientific applications. 


\section{THE MIDLEWARE ARCHITECTURE FOR THE REMOTE INSTRUMENTATION e-INFRASTRUCTURE}

\section{V.1. Overview and Basic Components of the Architecture}

The presented usage scenarios cover a diverse set of e-Science applications, from applications which provide Remote Instrumentation Services for real-time data acquisition and pre-processing to parallel applications which perform simulation and modelling based on acquired data. In order to meet all the application requirements to the e-Infrastructure and promote the joint research activities within the e-Science communities, the following middleware architecture is proposed for DORII (Fig. 1).

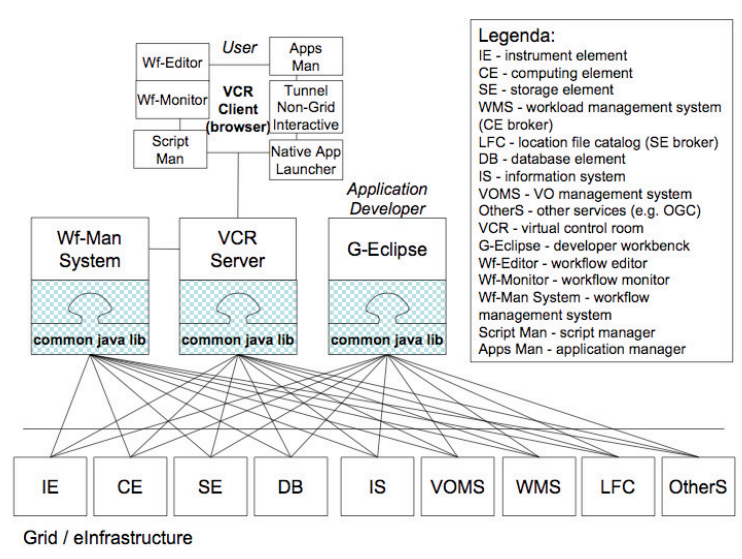

Fig. 1. The DORII middleware architecture

The architecture relies on gLite [26] that is deployed as a core middleware for EGEE shared resources, the DORII e-Infrastructure is based on. The high level gLite services cover main types of grid activities: authentication, authorization and updating entities (users, systems and services), provide information for post-mortem analysis of security-related events; provide functionality for data confidentiality and a dynamic connectivity service; providing a mechanism to publish and consume information by means of Information and Monitoring Services; using Job Monitoring Service and Network Performance Monitoring as well, etc.

The gLite middleware offers basic grid services such as Information, Job Management, Data Management and Security Services. Information about the resources and services of the infrastructure are provided by the Berkeley Database Information Index (BDII), which uses standard LDAP databases populated by an update process. The Workload Management System (WMS) is the service re- sponsible for the distribution and management of tasks across grid resources in such a way that applications are conveniently, efficiently and effectively executed. The Computing Element (CE) is responsible for submitting jobs to the underlying local cluster of Worker Nodes (WNs). Storage Elements (SEs) are responsible for data storage and management, while the LCG File catalogue (LFC) offers a hierarchical view of files to users with a UNIX-like client interface. From the security perspective the Virtual Organization Management Service (VOMS) is a full-fledged Attribute Authority whose job is to assign attributes like group membership and role ownership to members of a Virtual Organization (VO), so that other grid services can make informed decisions based on those attributes, with levels of granularity that range from extremely coarse to extremely fine.

Moreover, the functionality of the e-Infrastructure will be enhanced by middleware extensions dealing with the remote instrumentation management that are being built on top of the gLite stack. The users and the instruments interact through the Instrument Element (IE). The Instrument Element [26] is a concept initially developed by the GRIDCC project [11]. It is an abstraction of the instrument (or group of instruments) into a standard interface which can be used within the rest of the DORII architecture. The term instrument is used in this context to define a piece of equipment that needs to be initialized, configured, operated (start, stop, standby, resume, application specific commands), monitored or reset. The current version of the Instrument Element developed within the framework of DORII (IE2) is a set of WS-I compliant web services virtualizing the concept of instrument and sensor and presenting this as an e-Infrastructure component that works conjoined with the gLite middlware. The gLite security model is particularly used for providing the user authentication.

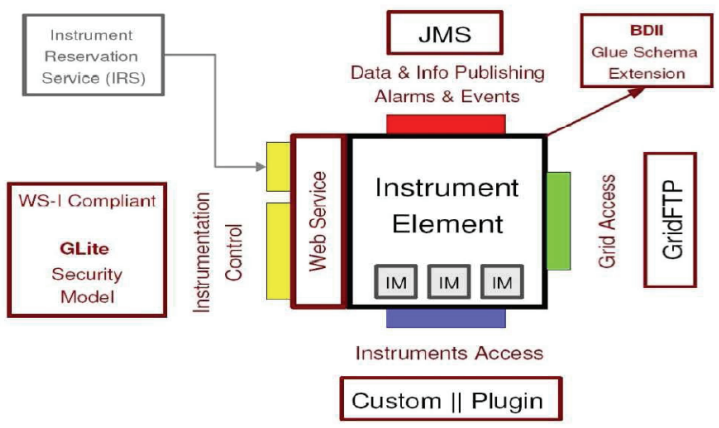

Fig. 2. The architecture of the IE2 component of the DORII infrastructure

The interface to a single instrument is presented in the architecture by the Instrument Manager (IM). IM is thereby a protocol adapter that allows the IE2 middleware to 
connect to the physical instrument (or its control system). A single IE can support multiple concurrent IMs (Fig. 2).

Furthermore, to deal with the interactivity requirements of the applications the DORII e-Infrastructure adopts a selection of middleware services deployed by the Int.EU.Grid Project. The derived services provide interactivity capabilities to the applications by introducing interactive agents (i2glogin and gvid [27]).

\section{V.2. Middleware Tools and Solutions Provided by the Architecture}

In order to fulfil the user requirements referring to the Remote Instrumentation Infrastructure, the architecture comprises several tools built on top of core services described in the previous section. The issues of the crosscomponent integration and collaboration between middleware tools and solutions within the common architecture will be handled by a specially deployed Common Library for DORII included to the architecture.

We present main tools outlining their main usage scenarios within the DORII e-Infrastructure in the following.

VCR [28] is a grid portal which gives registered users interactive access to all the DORII Grid resources and services, providing them with additional collaboration services like e-Logbook, video and audio conferences etc. VCR will support DORII applications with scripts and workflows through an external workflow management system (Wf-Man System), a native client application launcher and a tunnelling technology which can be used to integrate application components and to support interactivity. For this purpose the Wf-Man System of the VLab project will be used.

g-Eclipse [29] is an access and development platform for the DORII Grid and will be used whenever the application developers need an IDE for their sequential or parallel code. The g-Eclipse framework provides a set of tools for different grid actors. For the support of parallel applications special tracing and debugging tools will be provided by g-Eclipse [30].

Parallel applications using the Message-Passing Interface [31] cover an important class of the applications supported by DORII. In the frame of Int.EU.Grid, the MPI support was significantly improved compared with the general EGEE infrastructure. In this sense, important components of the Int.EU.Grid middleware that will be adopted in DORII are Open MPI, PACX-MPI and MPI-Start.

Open MPI [32] is an open-source state-of-the-art implementation of the complete MPI-2 standard. The team developing Open MPI is comprised of research and industrial partners with advanced knowledge of high-perform- ance computing and MPI implementations in particular. Open MPI is actively evolving and cooperating with different hardware and software vendors.

PACX-MPI [33] is an implementation of the MPI-1.2 (and parts of MPI-2) standard which focuses on running MPI applications on a metacomputer, possibly consisting of different heterogeneous hardware architectures, or in the context of a Grid, different clusters. For the local communication, the native MPI is used; for the inter-cluster communication, PACX-MPI handles all the communication details.

MPI-Start [34] was developed as a set of scripts in the frame of Int.EU.Grid. These scripts greatly improve running MPI applications for different MPI libraries, file systems and resource management systems in grid environments. It is also easy to extend the basic functionality by user routines.

\section{V.3. Workflow Management Solutions}

The main goal of the Workflow Manager System (WMS) is supporting users in definition, management and monitoring of measurement scenarios. What is outstanding (among other workflow system) in our solution is that it allows to connect many computational and experimental jobs in one execution graph.

The workflow component has been first introduced in the Virtual Laboratory project [35]. The first prototype of the WMS system has been deployed within the field of nuclear magnetic resonance spectroscopy and used by scientist to design and manage their experiment scenarios. The user was able to define experiment components, data flows between components, describe the experiment parameters and conditions. Moreover, the composed measurement scenario could be submitted for processing, i.e. to the grid environment.

The experience gained during the design and implementation of the VLab project is currently being used in the EXPReS project [36]. The overall objective of EXPReS is to create a production-level real-time, electronic VLBI, or e-VLBI, a service in which the radio telescopes are reliably connected to the central supercomputer at JIVE in the Netherlands via a high-speed optical-fibre communication network. The skeleton of the WMS has been used as a prototype of the application for controlling and managing data flows in the VLBI. The tool will be the central point of the VLBI, and will be used to design and control the observation.

Experiments executed in the science laboratories are usually complex and consist of many stages. Thus, we can define a graph which describes the execution path specified by a user. Nodes in this graph correspond to experimental, 
computational and storage tasks. Edges (links) correspond to the path the measurement execution is following. The type of application and its parameters are defined in nodes.

WMS facilitates the measurement process beginning from the preparation stage through experimental and computational processes to the result analysis (based on the achieved visualization data). WMS is functionally divided into three parts: editor, manager and monitor.

In Workflow Editor (WE) a user is given a list of building blocks. Each block represents the available resource. It is the user's responsibility to design and construct the experiment workflow from the available building blocks. However, the application can be equipped with the knowledge from the specific domain. In that case WE will be able to support users during the design phase of the workflow.

Each application available in the measurement scenario must be first analyzed from the functional point of view. Input and output parameters have to be taken into consideration. Also, input and output format files must be described. When the application analysis is done connection diagram must be prepared. This diagram consists of information on what possible paths between applications can be created.

The WE is designed as a stand alone application which can be launched using Java Web Start technology. Using Java Web Start technology, standalone Java software applications can be deployed with a single click over the network. Java Web Start ensures the most current version of the application will be deployed, as well as the correct version of the Java Runtime Environment will be used. The main advantage of such an approach is easy, unique and intuitive interface. Moreover, the application can be run at every computer connected to the Internet and equipped with a web browser.

Equipping the user with a graphical interface for a scenario definition has a big advantage - the user does not have to know the workflow description language. Using WE the user builds his/her workflow by connecting defined resources together and providing the required properties. After that workflow is submitted to the central management called Workflow Manager (WM), where is decomposed according to the user specification and each node is launched. All experimental tasks are directed to the Instrument Element (IE) service and similarly all computational tasks are submitted to the Computing Element (CE). Application results are stored on the Storage Element (SE).

Finally, WM needs grid infrastructure information which can be taken from the grid monitoring system. This information will be integrated with active workflow and presented to the user.
A general conception of the workflow work is presented in Fig. 3.

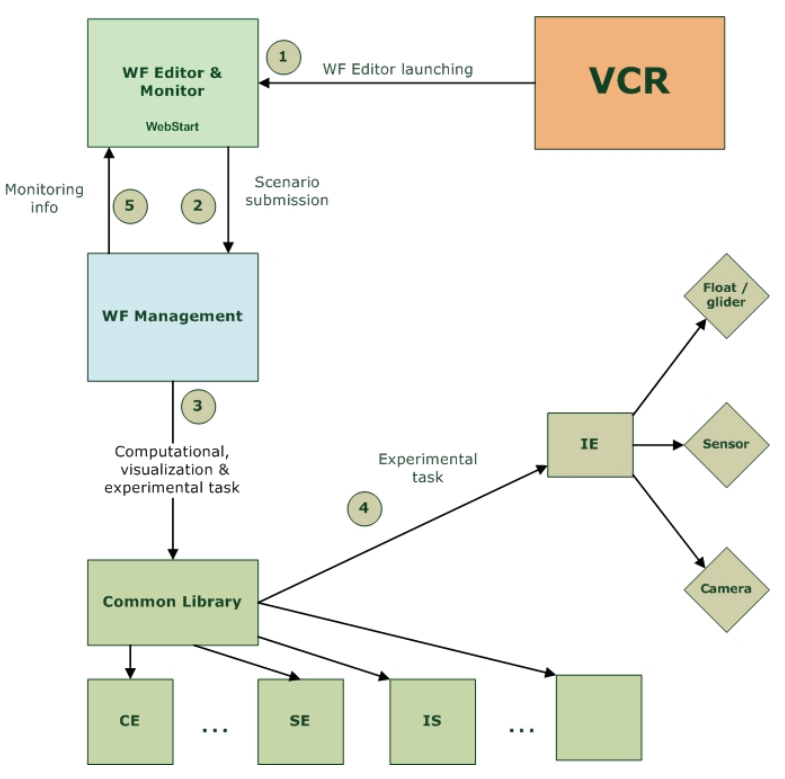

Fig. 3. Workflow conception

The concept of the WMS allows to define the process of an experiment in any way, from pre-processing, through executing the experiment to the post-processing and visualization tasks. Defining the measurement scenario allows to spare a lot of time during computation. The user does not have to wait for the end of a given process stage to submit another one. It is made automatically. Thanks to the Workflow Editor the user can easily define, submit and monitor the progress of the workflow realization.

\section{CONCLUSION}

The DORII project aims to establish a new e-Infrastructure that provides services identified in various applications operating remote instruments. Extending the level of scientific instruments' exploitation by integrating instruments and sensors into a grid computing environment, DORII will pave the way towards the deployment of the Remote Instrumentation Infrastructure for the operational support of applications using experimental laboratory equipment, especially where the time and resources needed for their running are considered impractical when using traditional IT infrastructures.

The e-Infrastructure is being promoted among the European and global research communities through the advanced software architecture which consolidates solutions expediting collaborative user activities on the Grid, extended and enhanced towards the full-service support of 
remote instrumentation resources and applications. The paper presented the architecture in terms of identified tools and services indispensable for the effective support of such applications by the e-Infrastructure.

The solutions and know-how developed and enhanced in DORII might be of interest also to other e-Science communities. The project aims at spreading out these innovations.

\section{Acknowledgments}

This research is supported by the European Commission within the 7th Framework Program (FP7/2007-2013) under grant agreement no. RI-213110.

\section{References}

[1] Enabling Grids for E-Science (EGEE) project website, http://www.eu-egee.org/

[2] Distributed European Infrastructure for Supercomputing Applications (DEISA) project website, http://www.deisa.eu/

[3] Open Science Grid (OSG) project website, http://opensciencegrid.org/

[4] National Research Grid Initiative (NAREGI) project website, http://www.naregi.org/index_e.html

[5] D. Roure, N. Jennings and Shadbolt, Research Agenda for the Semantic Grid: A Future e-Science Infrastructure. 2001, http://www.semanticgrid.org/v1.9/semgrid.pdf

[6] V. J. Harward et al., The iLab shared architecture: A Web Services infrastructure to build communities of Internet accessible laboratories. Proc. IEEE 96 (6), 931-950 (2008).

[7] D. F. McMullen, R. Bramley, K. Chiu, H. Davis, T. Devadithya, J. C. Huffman, K. Huffman and T. Reichherzer, The Common Instrument Middleware Architecture. In: F. Davoli, N. Meyer, R. Pugliese and S. Zappatore, Eds., Grid Enabled Remote Instrumentation. Springer, New York, NY, 2008, pp. 393-407.

[8] F. Lelli, E. Frizziero, M. Gulmini, G. Maron, S. Orlando, A. Petrucci and S. Squizzato, The many faces of the integration of instruments and the grid. International Journal of Web and Grid Services 3 (3), 239-266 (2007).

[9] F. Davoli, S. Palazzo and S. Zappatore, Distribute Cooperative Laboratories: Networking, Instrumentation, and Measurements. Springer, New York, 2006.

[10] RINGrid project website, http://www.ringrid.eu/

[11] GRIDCC project website, http://www.gridcc.org/

[12] The Common Instrument Middleware Architecture (CIMA) project website, http://www.instrumentmiddleware.org/

[13] Deployment of Remote Instrumentation Infrastructure (DORII) project website, http://www.dorii.eu/

[14] I. Foster, C. Kesselman, J. M. Nick and S. Tuecke, The Physiology of the Grid - An Open Grid Services Architecture for Distributed Systems Integration. http://www.globus.org/alliance/publications/papers/ogsa.pdf

[15] Interactive European Grid (Int.EU.Grid) project website, http://www.i2g.eu/
[16] H. Kornmayer, M. Stümpert, M. Knauer and P. Wolniewicz, g-Eclipse - an Integrated Workbench Tool for Grid application users, Grid operators and Grid application developers. Cracow Grid Workshop '06, Cracow, Poland, October $15-18,2006$.

[17] M. Okon, D. Kaliszan, M. Lawenda, D. Stokłosa, T. Rajtar, N. Meyer and M. Stroinski, Virtual Laboratory as a Remote and Interactive Access to the Scientific Instrumentation Embedded in Grid Environment. Proceedings of the Second IEEE International Conference on e-Science and Grid Computing (e-Science'06)

http://ieeexplore.ieee.org/stamp/stamp.jsp?arnumber=04031097

[18] GEANT2 project website, http://www.geant2.net/

[19] Open Grid Forum (OGF) website, http://www.ogf.org/

[20] K. Keahey, M. E. Papka, Q. Peng, D. Schissel, G. Abla, T. Araki, J. Burruss, Feibush, P. Lane, S. Klasky, T. Leggett, D. McCune and L. Randerson, Grids for Experimental Science: The Virtual Control Room. Proceedings of the Second International Workshop on Challenges of Large Applications in Distributed Environments (CLADE'04), http://ieeexplore.ieee.org/iel5/9148/29051/01309087.pdf

[21] I. Foster, C. Kesselman and S. Tuecke, The Anatomy of the Grid: Enabling Scalable Virtual Organizations. Intl. J. Supercomputer Applications, 2001. www.globus.org/research/papers/anatomy.pdf

[22] R. H. Menk, L. Rigon and F. Arfelli, Diffraction-enhanced $X$-ray medical imaging at the ELETTRA synchrotron light source. Nuclear Instruments and Methods in Physics Research A548, 213-220 (2005).

[23] European Centre for Training and Research in Earthquake Engineering (EUCENTRE) website, http://www.eucentre.it/

[24] A. Crise, P. Lazzari, S. Salon and A. Teruzzi, MERSEA deliverable D11.2.1.3 - Final report on the BFM OGSOPA Transport module. 21 pp., 2008.

[25] E. Laure, S. M. Fisher, A. Frohner, C. Grandi, P. Kunszt, A. Krenek, O. Mulmo, F. Pacini, F. Prelz, J. White, M. Barroso, P. Buncic, F. Hemmer, A. Di Meglio and A. Edlund, Programming the Grid with gLite. Computational Methods In Science And Technology 12 (1), 33-45 (2006).

[26] E. Frizziero, M. Gulmini, F. Lelli, G. Maron, A. Oh, S. Orlando, A. Petrucci, S. Squizzato and S. Traldi, Instrument Element: a new Grid component that enables the control of remote instrumentation. Proc. 6th IEEE Internat. Symp. on Cluster Computing and the Grid Workshops (CCGRIDW'06), http://ieeexplore.ieee.org/ie15/10857/34198/01630943.pdf.

[27] H. Rosmanith and D. Kranzlmueller, Glogin - A Multifunctional, Interactive Tunnel into the Grid. In: Proceedings of the 5th IEEE/ACM International Workshop on Grid Computing, Pittsburgh, PA, USA, November 2004.

[28] R. Ranon, L. De Marco, A. Senerchia, S. Gabrielli, L. Chittaro, R. Pugliese , L. Del Cano, F. Asnicar and M. Prica, A Web-based Tool for Collaborative Access to Scientific Instruments in Cyberinfrastructures. In: F. Davoli, N. Meyer, R. Pugliese and S. Zappatore, Eds., Grid Enabled Remote Instrumentation, Springer, New York, NY, 237251 (2008)

[29] H. Kornmayer, M. Stümpert, M. Knauer and P. Wolniewicz, g-Eclipse - an Integrated Workbench Tool for Grid application users, Grid operators and Grid application developers. Cracow Grid Workshop '06, Cracow, Poland, October 15-18, 2006.

[30] C. Klausecker, T. Köckerbauer, R. Preissl and D. Kranzlmüller, Debugging MPI Programs on the Grid using g- 
Eclipse. 2nd Parallel Tools Workshop, Stuttgart, Germany, July 7-8, 2008.

[31] J. J. Dongarra, S. W. Otto, M. Snir and D. Walker, A message passing standard for MPP and workstations. Communications of the ACM, 39 (7), 84-90 (1996).

[32] Open MPI website, http://www.open-mpi.org/

[33] R. Keller and M. Liebing, Using PACX-MPI in MetaComputing applications. 18-th Symposium Simulationstechnique, Erlangen, Sept. 12-15, 2005.
[34] MPI-Start website, http://www.hlrs.de/organization/amt/projects/mpistart/

[35] Virtual Laboratory (VLAB) project website, http://vlab.psnc.pl/

[36] EXPReS project website, http://www.expres-eu.org/

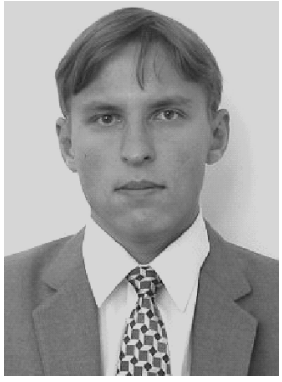

Dr. AleXey Cheptsov is a research scientist at the High-Performance Computing Center Stuttgart (Germany). He attained his M.Sc. degree in Computer Science in 2002 at the National University of Technology of Donetsk (Ukraine) and his Ph.D. in 2007 at the Research Institute of Simulation Problems in Power Engineering, National Academy of Science of Ukraine. Throughout his scientific career he has conducted research and published in areas of simulation support of complex dynamic systems, deployment of technologically-oriented distributed simulation environments for diverse problem areas and parallel and highperformance simulation technology. His current research interests include providing Grid support for scientific applications, improvement of application communication patterns and parallel communication libraries. He is currently involved in the DORII project.
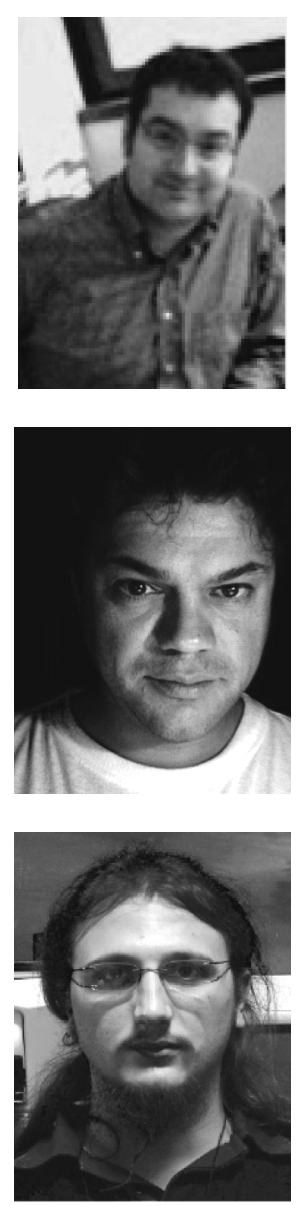

Milan Prica is a senior software engineer working for the Software for Measurements Group of Sincrotrone Trieste. His background is on Computer Science, Information Retrieval and Search Engine technologies. His interests include Grid Systems, Data Management, Reliable Systems and Web 2.0. Major projects that he has participated in include ADAPT, GRIDCC and DORII.

Dr. Roberto Pugliese is a research coordinator at Sincrotrone Trieste S.C.p.A. where he is leading the Scientific Computing Group. Since October 2002 he has also been Professor of E-Commerce at the University of Udine. His research interests include Web Based Virtual Collaborations and Grid technologies. Roberto Pugliese was the technical coordinator of the GRIDCC project and is currently coordinating the Applications workpackage of the DORII project.

ANDREA DEL LiNZ is a software engineer specialized in OO programming and Web development in Sincrotrone Trieste. His interests are on Robotics, Grid Infrastructures, and Remote Instrumentation. He has participated in the GRIDCC and DORII projects. 

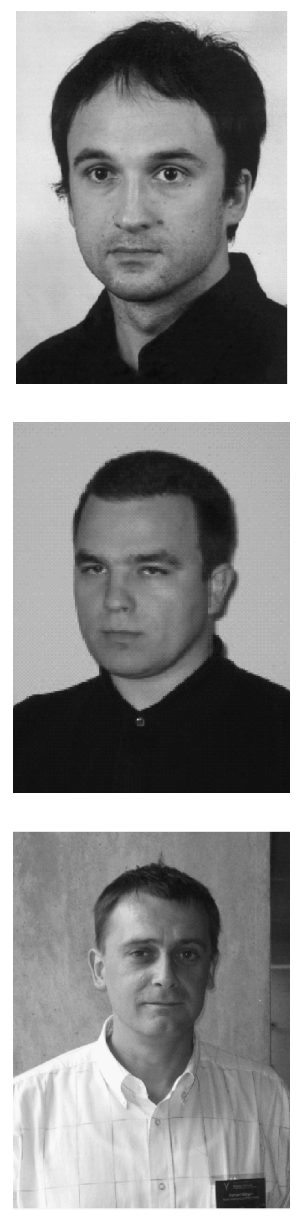

Marcin Plóciennik (MP) received the M.Sc. degree in Computer Science from the Poznań University of Technology in 1999. Currently he is working at the Supercomputing Department in PSNC. He is Deputy Project Coordinator of the FP7 DORII project. His research interests concern distributed computing systems, Grid environments, web technologies and technology of graphical user interfaces development. From 1998 to 2002 MP was taking part in an international project of GUI development for mobile network analysts. Working in PSNC since 2002, he participated in several national and international projects e.g. the CrossGrid, PROGRESS, BalticGrid, Int.EU.Grid, Euforia, DORII. He is also author and co-author of several papers and reports.

MARCin LAWENDa graduated from the Poznań University of Technology and received M.Sc. in Computer Science (Parallel and Distributed Computation area) in 2000. He defended the Ph.D. thesis at the same university at Faculty of Computing Science and Management, Institute of Computing Science, in 2006. He currently works for Poznan Supercomputing and Networking Center on the project manager position on the Virtual Laboratory project. His research interests include parallel and distributed computing, scheduling and Grid technologies. He has been a member of the Polish Information Processing Society since 2000.

Dr. NORBert MeYer is currently the head of the Supercomputing Department in Poznan Supercomputing and Networking Center (http://www.man.poznan.pl ). His research interests concern resource management in GRID environment, GRID accounting, data management, technology of development graphical user interfaces and network security, mainly in the aspects of connecting independent, geographically distant Grid domains. He conceived the idea of connecting Polish supercomputing centres, a vision of dedicated application servers and distributed storage infrastructure. He is the author and co-author of $60+$ conference papers and articles in international journals, member of programme committees of international conferences related high performance computing and grid computing. Norbert Meyer was the leader of RINGrid EU project, currently leading the DORII project. 